\title{
ZnGa2O4:Mn2+ phosphors grown by Laser Floating Zone
}

\author{
N. F. Santos, J. Rodrigues, C. Nico, A. J. S. Fernandes, F. M. Costa and T. Monteiro \\ Department of Physics, Laboratory I3N, University of Aveiro, Campus de Santiago, 3810-193 Aveiro, \\ Portugal
}

Cubic zinc gallate $\left(\mathrm{c}-\mathrm{ZnGa}_{2} \mathrm{O}_{4}\right)$ has attracted the attention of the scientific community due to its potential phosphor applications [1-6], namely in field emission displays (FEDs) and other electroluminescent devices. Among other advantages, this oxide matrix shows superior thermal and chemical stability when compared to $\mathrm{ZnS}$ based phosphors [1]. Most of the above mentioned works comprise nanostructures, thin films or pressed pellets while scarce information is found on bulk c$\mathrm{ZnGa}_{2} \mathrm{O}_{4}$ material. In particular, no records were found regarding $\mathrm{c}-\mathrm{ZnGa}_{2} \mathrm{O}_{4}$ crystal growth by the laser floating zone (LFZ) technique. In this work, crystalline fibres of manganese doped $(0.01 \mathrm{~mol} \%)$ zinc gallate were produced via LFZ in order to investigate its applicability in efficient phosphors. The transition metal ions are suitable activators and show some advantages over the widely used rare earths, namely at environmental and economic levels.

The secondary electron image of the fibre's longitudinal section (figure 1) reveals a polycrystalline and polyphasic nature, as corroborated by powder X-ray diffraction measurements (figure 2), from which the presence of gallium oxide and zinc oxide phases is inferred, in addition to the zinc gallate phase. The room temperature samples' green luminescence obtained with $325 \mathrm{~nm}$ photon excitation (figure 3, left side) is activated by the manganese ions in the zinc gallate host. Under these excitation conditions, the low temperature photoluminescence (PL) spectrum (figure 3, right side) reveals that the intraionic $\mathrm{Mn}^{2+}$ emission is due to an overlap of $\mathrm{Mn}^{2+}$ centres in different symmetry environments, as expected from the polycrystalline nature of the fibres. The transition metal ions incorporated in high site symmetry, at tetrahedral sites (substituting the zinc ions in the c- $\mathrm{ZnGa}_{2} \mathrm{O}_{4}$ lattice), are preferentially excited despite the important contribution from centres located at sites with lower symmetry.

Further work is underway after observation of a strong room temperature emission from the zinc gallate phase at the molten zone in order to obtain monocrystalline zinc gallate fibres and therefore optimize the manganese emission in this host.

\section{References}

1. B. Liu et al., Nanotechnology 20: 365705, 2009

2. I. J. Hsieh et al., J. Appl. Phys. 76: 3735-3739, 1994

3. V. Singh et al., J. Lumin. 131: 1789-1794, 2011

4. D. P. Dutta et al., J. Phys. Chem. C 113: 16954-16961, 2009

5. J. S. Kim et al., Mater. Lett. 58: $1354-1357,2004$

6. T.K. Tran et al., J. Appl. Phys. 78: 5691-5695, 1995

The authors acknowledge FCT for the financial funding from PTDC/CTM/100756/2008 and PEstC/CTM/LA0025/2011 projects. 


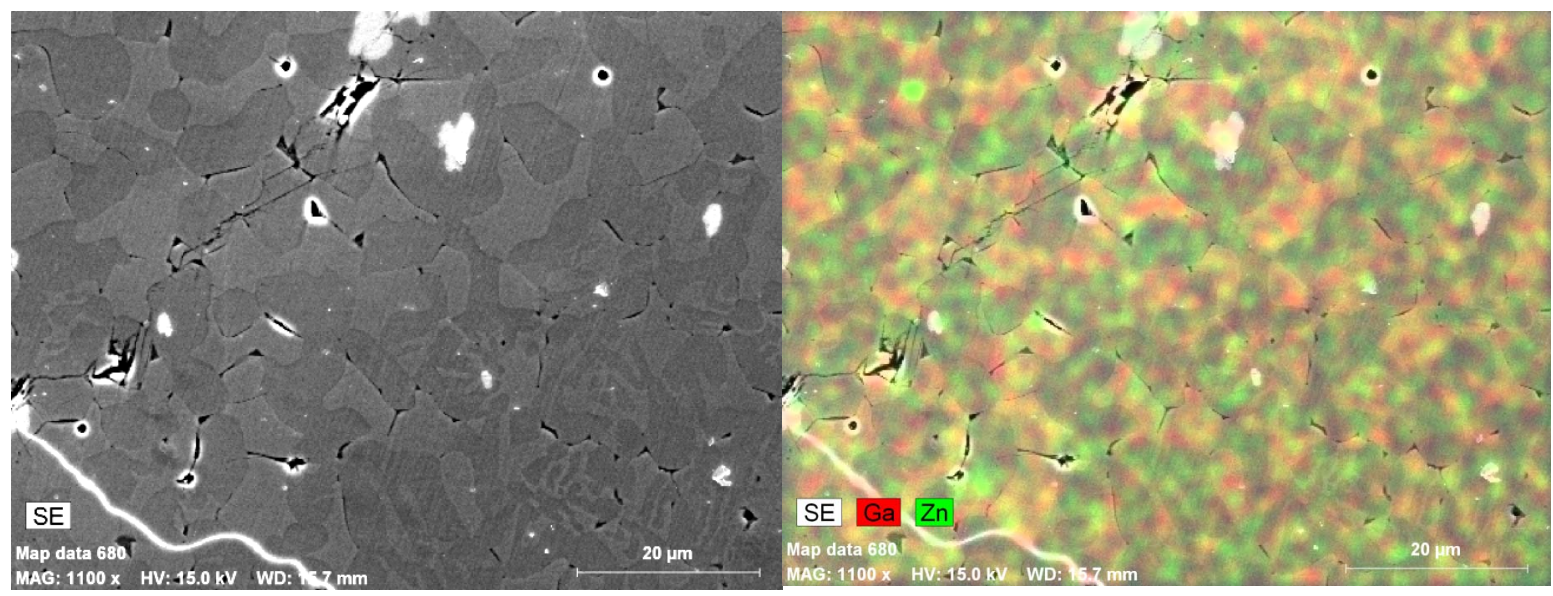

Figure 1. Secondary electron image of a zinc gallate fibre's polished longitudinal section and its superposition with an energy dispersive X-ray spectroscopy mapping.

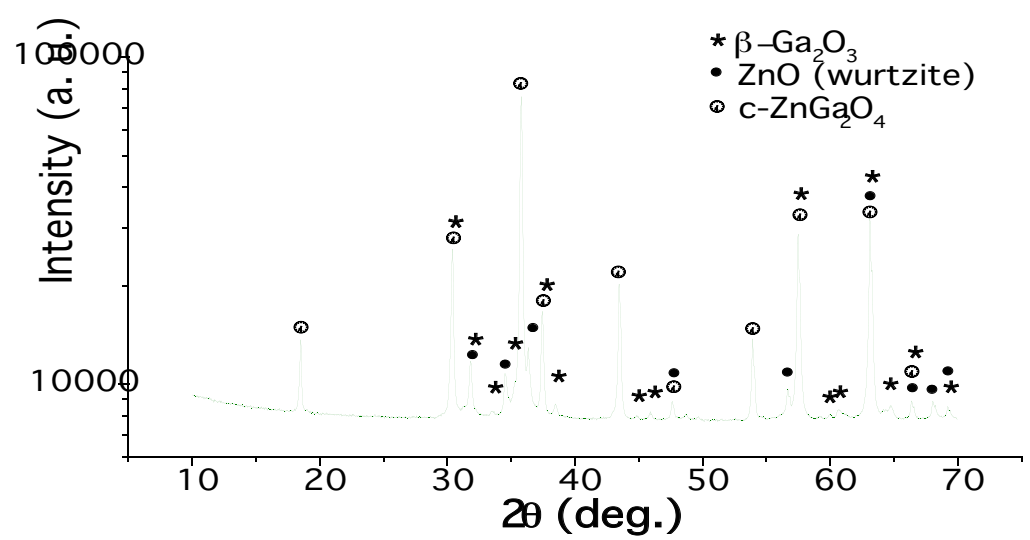

Figure 2. Powder X-ray diffraction pattern of the LFZ grown zinc gallate fibre.
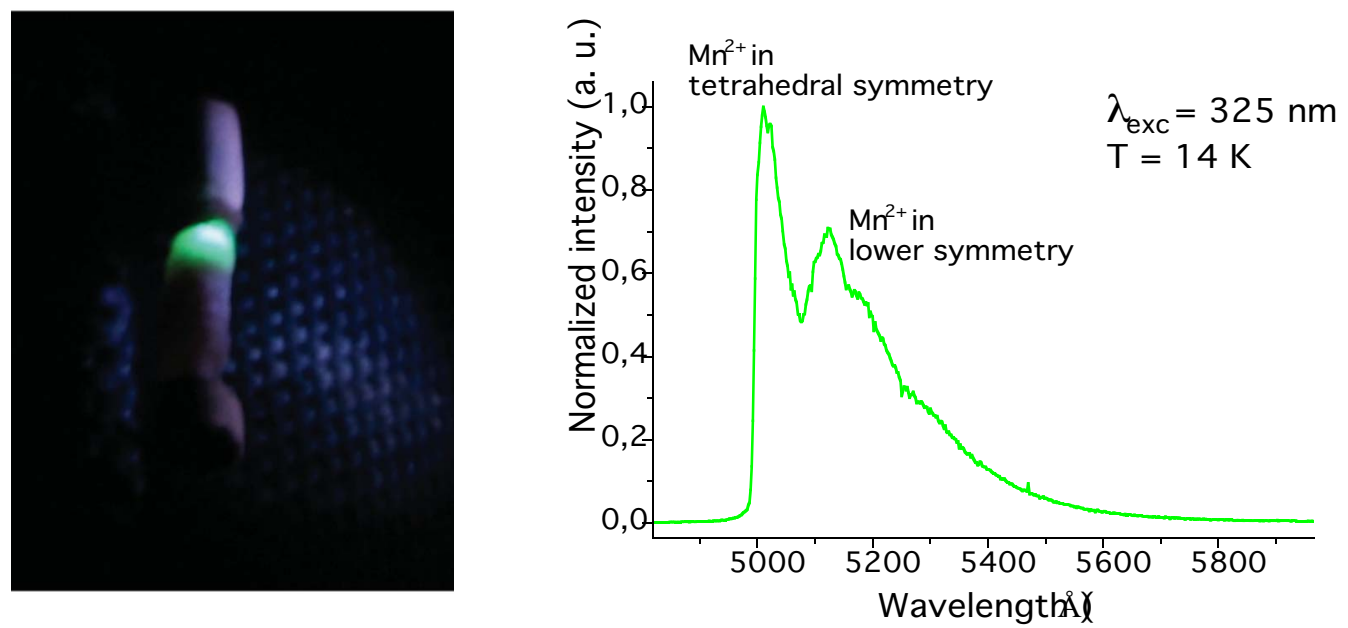

Figure 3. Room temperature luminescence of the zinc gallate fibre obtained with $325 \mathrm{~nm}$ photon excitation, and PL spectrum taken at $14 \mathrm{~K}$ under the same excitation conditions. 